\title{
DERIVED LENGTH OF ZERO ENTROPY GROUPS ACTING ON PROJECTIVE VARIETIES IN ARBITRARY CHARACTERISTIC-A REMARK TO A PAPER OF DINH-OGUISO-ZHANG
}

\author{
SICHEN LI
}

\begin{abstract}
Let $X$ be a projective variety of dimension $n \geq 1$ over an algebraically closed field of arbitrary characteristic. We prove a Fujiki-Lieberman type theorem on the structure of the automorphism group of $X$. Let $G$ be a group of zero entropy automorphisms of $X$ and $G_{0}$ the set of elements in $G$ which are isotopic to the identity. We show that after replacing $G$ by a suitable finite-index subgroup, $G / G_{0}$ is a unipotent group of the derived length at most $n-1$. This result was first proved by Dinh, Oguiso and Zhang for compact Kähler manifolds.
\end{abstract}

\section{INTRODUCTION}

Let $X$ be a projective variety of dimension $n \geq 1$ over an algebraically closed field $k$ of arbitrary characteristic. It is well known that the automorphism group scheme Aut $_{X}$ of a projective variety $X$ is locally of finite type over $k$ and $\operatorname{Aut}(X)=\operatorname{Aut}_{X}(k)$; in particular, the reduced neutral component $\left(\mathrm{Aut}_{X}^{0}\right)_{\text {red }}$ of $\mathrm{Aut}_{X}$ is a smooth algebraic group over $k$ (cf. $[2, \S 7])$. Denote $\left(\operatorname{Aut}_{X}^{0}\right)_{\text {red }}(k)$ by $\operatorname{Aut}_{0}(X)$. If $G$ is a subgroup of $\operatorname{Aut}(X)$, define

$$
G_{0}:=G \cap \operatorname{Aut}_{0}(X)
$$

Denote by $\mathrm{NS}(X)=\operatorname{Pic}(X) / \operatorname{Pic}^{0}(X)$ the Néron-Severi group of $X$, i.e., the finitely generated abelian group of Cartier divisors on $X$ modulo algebraic equivalence. For a field $\mathbf{F}=$ $\mathbf{Q}, \mathbf{R}$ or $\mathbf{C}$, the $\mathbf{F}$-vector space $\mathrm{NS}_{\mathbf{F}}(X)$ stands for $\mathrm{NS}(X) \otimes_{\mathbf{Z}} \mathbf{F}$; it is a finite-dimensional $\mathbf{F}$-vector space. Define the first dynamcial degree of an automorphism $g \in \operatorname{Aut}(X)$ as the spectral radius of its natural action $g^{*}$ on $\mathrm{NS}_{\mathbf{R}}(X)$, i.e.,

$$
d_{1}(g):=\rho\left(\left.g^{*}\right|_{\mathrm{NS}_{\mathbf{R}}(X)}\right):=\max \left\{|\lambda|: \lambda \text { is an eigenvalue of }\left.g^{*}\right|_{\mathrm{NS}_{\mathbf{R}}(X)}\right\} .
$$

We say that $g$ is of positive entropy if $d_{1}(g)>1$, otherwise it is of zero entropy. We call $G$ of positive entropy, if every element of $G \backslash\{\mathrm{id}\}$ is of positive entropy.

2010 Mathematics Subject Classification. 14G17, 14J50, 37B40, 14 C25.

Key words and phrases. positive characteristic, automorphism of varieties, dynamics, zero entropy, unipotent group, derived length.

The author was partially supported by the China Scholar Council 'High-level university graduate program'. 
For a subgroup $G$ of the automorphism group $\operatorname{Aut}(X)$, we define the zero-entropy subset of $G$ as

$$
N(G):=\left\{g \in G: g \text { is of zero entropy, i.e., } d_{1}(g)=1\right\} .
$$

We call $G$ of zero entropy, if $N(G)=G$. For the study of dynamical degrees, we refer to [8, $\S 4]$ as a survey and $[4,21,12]$ in arbitrary characteristic.

It is known that $\operatorname{Aut}(X)$ satisfies a Tits alternative and for solvable subgroups of $\operatorname{Aut}(X)$, the positive entropy part has a "bounded" size. Precisely, the following result of the case of compact Kähler manifolds or complex projective varieties with mild singularities was proved in [23,3], and of the case of projective varieties in arbitrary characteristic was proved in [12].

Theorem 1.1. Let $X$ be a projective variety of dimension $n \geq 1$ and $G$ a subgroup of $\operatorname{Aut}(X)$. Suppose that $G$ does not contain any non-abelian free subgroup. Then there is a finite-index subgroup $G^{\prime}$ of $G$ such that the quotient group $G^{\prime} / N\left(G^{\prime}\right)$ is a free abelian of rank $r \leq n-1-\max \left\{0, \kappa(X), \kappa\left(\omega_{X^{v}}\right)\right\}$. Here, $\kappa(X)$ and $\kappa\left(\omega_{X^{v}}\right)$ denote the Kodaira dimension of $X$ and the Kodaira-Iitaka dimension of normalization $X^{v}$ of $X$.

For the definitions of $\kappa(X)$ and $\kappa\left(\omega_{X^{v}}\right)$, we refer to [19, Appendiexs A and B] or [12, $\S 2.1 .1]$. When char $k=0$, we know that $\kappa(X)=\kappa\left(\omega_{X^{v}}\right)=\kappa(\tilde{X})$, where $\widetilde{X} \rightarrow X$ is a projective resolution.

If $X$ admits a group $G$ such that the rank of $G^{\prime} / N\left(G^{\prime}\right)$ is maximal, i.e., equal to $n-1$, we will say that $X$ is a variety with maximal dynamical rank (MDR for short). Clearly, for such a variety, we have $\kappa(X) \leq n-1-\operatorname{rank}\left(G^{\prime} / N\left(G^{\prime}\right)\right)=0$ by Theorem 1.1. We refer to [7, 24] for more properties of these varieties over complex number field $\mathrm{C}$. The problem of classifying variety with MDR is still open when either char $k>0$ or $X$ is rational connected. In [11], the authors tried to characterize the complex projective varieties of sub-maximal dynamical rank, i.e., equal to $n-2$.

In the note, we will focus our study on the group $N\left(G^{\prime}\right)$ in the last statement. In order to simplify the notation, we consider groups $G$ such that every element of $G$ is of zero entropy.

Now recall that for a group $G$ and a non-negative integer $l$, the $l$-th derived series $G^{(l)}$ is defined inductively by

$$
G^{(0)}:=G \quad \text { and } \quad G^{(i+1)}:=\left[G^{(i)}, G^{(i)}\right] .
$$

By definition, $G^{(l)}=\{1\}$ for some non-negative integer $l$ exactly when $G$ is solvable. We call the minimum of such $l$ the derived length of $G$ (when $G$ is solvable) and denote it by

$$
\ell(G) \text {. }
$$

A group $H$ is said to be unipotent if there is an injective homomorphism $\rho: H \rightarrow \operatorname{GL}(N, \mathbf{R})$ such that for every $h \in H$, the image $\rho(h)$ is upper triangular with all entries on the diagonal being 1 . Note that unipotent groups are solvable. It is known that if a group $H$ is isomorphic 
to a subgroup of $\operatorname{GL}(N, \mathbf{R})$ whose elements have only eigenvalue 1 , then $H$ is unipotent, see $[13, \S 17.5]$. Below is our first result which slightly extends [6, Theorem 1.2].

Theorem 1.2. Let $X$ be a projective variety of dimension $n \geq 1$ and $G$ a subgroup of $\operatorname{Aut}(X)$ such that every element of $G$ is of zero entropy. Then

(1) $G$ admits a finite-index subgroup $G^{\prime}$ such that, for any $1 \leq k \leq n-1$, the natural map $G^{\prime} /\left.G_{0}^{\prime} \longrightarrow G^{\prime}\right|_{N_{\mathbf{R}}^{k}(X)}$ is an isomorphism with image a unipotent subgroup of $\mathrm{GL}\left(N_{\mathbf{R}}^{k}(X)\right)$.

(2) For every finite-index subgroup $G^{\prime}$ of $G$ such that $G^{\prime} / G_{0}^{\prime}$ is a unipotent group, the derived length of $G^{\prime} / G_{0}^{\prime}$ does not depend on the choice of $G^{\prime}$ and is at most equal to $n-1$.

Remark 1.3. (1) After replacing $H^{k, k}(X, \mathbf{F}), \mathcal{K}_{i}(X)$, Kähler cone,etc as in [6] by $N_{\mathbf{F}}^{k}(X)$, $\operatorname{Nef}^{i}(X)$, ample cone,etc respectively, this proof in Theorem 1.2 uses the same argument in [6]. We will give a sketch of the proof in section 2. The main techniques used in this section are a Fujiki-Lieberman type theorem (cf. Theorem 1.4 below) and a higher-dimensional Hodge-index theorem for R-Cartier divisors (cf. [12, Proposition 2.9]).

(2) In [6], Dinh, Oguiso and Zhang further established that

$$
\ell\left(G^{\prime} / G_{0}^{\prime}\right) \leq n-\max \{\kappa(X), 1\}
$$

for a compact Kähler manifold $X$. It is hard to generalize this result in arbitrary characteristic. Indeed, their argument essentially depends on the Deligne-Nakamura-Ueno theorem (cf. [17, Corollary 2.4]), which is not known in positive characteristic, as far as we know.

Now let $X$ be a complex normal projective variety and $B$ a Cartier divisor on $X$. Denote by $\operatorname{Aut}_{[B]}(X):=\left\{g \in \operatorname{Aut}(X) \mid g^{*}[B]=[B]\right\}$. When $X$ is smooth and $B$ is ample, Fujiki and Lieberman proved in [9, Theorem 4.8] and [14, Proposition 2.2] that $\left[\operatorname{Aut}_{[B]}(X)\right.$ : $\left.\operatorname{Aut}_{0}(X)\right]<\infty$. Generally, let $G$ be a subgroup of $\operatorname{Aut}(X)$, such that for any $g \in$ $G, g^{*}\left[B_{g}\right]=\left[B_{g}\right]$ for some big Cartier divisor $B_{g}$. Dinh, Hu and Zhang proved in [5, Theorem 2.1] that $G$ is virtually in $\operatorname{Aut}_{0}(X)$, i.e., $\left[G: G \cap \operatorname{Aut}_{0}(X)\right]<\infty$. After replacing $g^{*}\left[B_{g}\right]=\left[B_{g}\right]$ by $g^{*} B_{g} \equiv_{w} B_{g}$ for some big Weil R-divisor $B_{g}$, Meng and Zhang showed in [15, Theorem 1.2] that $G$ is virtually in $\operatorname{Aut}_{0}(X)$, where $" \equiv_{w} "$ is the weak numerical equivalence (cf. [15, Definition 2.2]). Using a Hilbert scheme argument, Meng and Zhang proved in [16, Remark 2.6] that for an ample divisor $H$ on a projective variety $X$ in arbitrary characteristic, $\left[\operatorname{Aut}_{[H]}(X): \operatorname{Aut}_{0}(X)\right]<\infty$.

In the note, we can further generalize [15, Theorem 1.2] to the following.

Theorem 1.4. Let $X$ be a projective variety. Let $G$ be a subgroup of $\operatorname{Aut}(X)$, such that for any $g \in G, g^{*}\left[B_{g}\right]=\left[B_{g}\right]$ for some big $\mathbf{R}$-divisor $B_{g}$ in $N_{\mathbf{R}}^{1}(X)$. Then $G$ is virtually in $\operatorname{Aut}_{0}(X)$, i.e., $\left[G: G \cap \operatorname{Aut}_{0}(X)\right]<\infty$. 
Notation and Terminology. We recall the definitions of $N_{\mathbf{R}}^{k}(X)$ and $\operatorname{Nef}^{k}(X)$ in [10]. $N_{\mathbf{Z}}^{k}(X)$ (or $N_{k}(X)_{\mathbf{Z}}$ ) is the group of codimension (or dimension) $k$ algebraic cycles on $X$ modulo numerical equivalence. We will use the vector spaces $N_{\mathbf{R}}^{k}(X):=N_{\mathbf{Z}}^{k}(X) \otimes_{\mathbf{Z}}$ $\mathbf{R}, N_{\mathbf{C}}^{k}(X):=N_{\mathbf{Z}}^{k}(X) \otimes_{\mathbf{Z}} \mathbf{C}$ and $N_{k}(X)_{\mathbf{R}}:=N_{k}(X)_{\mathbf{z}} \otimes_{\mathbf{Z}} \mathbf{R}$. A $k$-cycle $Z$ is effective, if all of its defining coefficients are non-negative. The corresponding numerical class $[Z] \in N_{k}(X)_{\mathbf{R}}$ is called an effective numerical class. We denote by $\overline{\mathrm{Eff}}_{k}(X)$ the closure of the convex cone generated by all effective numerical classes in $N_{k}(X)_{\mathbf{R}}$. It is called the pseudo-effective cone of $N_{k}(X)_{\mathbf{R}}$. The cone dual to $\overline{\operatorname{Eff}}_{k}(X)$ in $N_{\mathbf{R}}^{k}(X)$ is called the nef cone $\operatorname{Nef}^{k}(X)$, which is a salient closed convex cone of full dimension (i.e., it generates $N_{\mathbf{R}}^{k}(X)$ as a vector space). An element of $\operatorname{Nef}^{k}(X)$ is called a nef class. In particular, $\operatorname{Nef}^{1}(X)$ is the usual nef cone $\operatorname{Nef}(X)$ consisting of all nef $\mathbf{R}$-Cartier divisor classes.

Now let $X$ and $G$ be as in Theorem 1.2 and $H$ a finite-index solvable subgroup of $G$. We quote $\ell_{\text {ess }}(G, X)$ and $\ell_{\min }(H)$ in $[6, \S 1]$. The essential length of the action of $G$ on $X$ is defined by

$$
\ell_{\text {ess }}(G, X):=\ell\left(G^{\prime} / G_{0}^{\prime}\right) .
$$

Here, $G^{\prime}$ is any finite-index subgroup of $G$ such that $G^{\prime} / G_{0}^{\prime}$ is a unipotent group. This definition does not depend on the choice of $G^{\prime}$, see [6, Lemma 2.7] or Lemma 2.1 below. We also define

$$
\ell_{\min }(H):=\min _{H^{\prime}} \ell\left(H^{\prime}\right)
$$

Here, $H^{\prime}$ runs through all finite-index solvable subgroups of $H$, see also Lemma 2.1.

When a group $G$ acts on a space $V$, we denote by $\left.G\right|_{V}$ the image of the canonical homomorphism $G \rightarrow \operatorname{Aut}(V)$. For instance, $\left.\operatorname{Aut}(X)\right|_{N_{\mathbf{R}}^{k}(X)}$ is the image of the canonical action of the automorphism group $\operatorname{Aut}(X)$ on $N_{\mathbf{R}}^{k}(X)$. For a normal subgroup $G_{1} \unlhd G$, we set $\left.\left(G / G_{1}\right)\right|_{V}=\left(\left.G\right|_{V}\right) /\left(\left.G_{1}\right|_{V}\right)$. If $L$ and $M$ are two numerical classes, we denote by $L \cdot M$ or $L M$. We also identify $N_{\mathbf{R}}^{0}(X)$ and $N_{\mathbf{R}}^{n}(X)$ with $\mathbf{R}$ in the canonical way. So classes in these groups are identified to real numbers. For a linear map $f: V \rightarrow V$, we denote by $\|f\|$ the norm of $f$.

\section{Proof of Theorems 1.2 AND 1.4}

We first quote two lemmas in [6], which will be used in the proof of Theorem 1.2.

Lemma 2.1. (cf. [6, Lemma 2.7]) Let $H$ be a unipotent group and let $H^{\prime}$ be a finite-index subgroup of $H$. Then we have $\ell\left(H^{\prime}\right)=\ell(H)$. In particular, we have $\ell_{\min }(H)=\ell(H)$.

Lemma 2.2. (cf. [6, Lemma 2.1]) Let $V$ be a real vector space of finite dimension. Let $\Gamma$ be a subgroup of $\mathrm{GL}(V)$. Assume there is an integer $N \geq 1$ such that $g^{N}$ is unipotent for every $g \in \Gamma$, i.e., their eigenvalues are 1. Then there is a finite-index subgroup $\Gamma^{\prime}$ of $\Gamma$ which is a unipotent subgroup of $\mathrm{GL}(V)$.

To prove Theorem 1.4, we needs a crucial lemma in [15]. 
Lemma 2.3. ( $c f$. [15, Proposition 2.9]) Let $f: V \rightarrow V$ be an invertible linear map of a positive dimensional real normed vector space $V$ such that $f^{ \pm 1}(C)=(C)$ for a closed convex cone $C \subseteq V$ which spans $V$ and contains no line. Let $q$ be a positive number. Then (1) and (2) below are equivalent.

(1) $f(x)=q x$ for some $x \in C^{\circ}$ ( the interior part of $C$ ).

(2) There exists a constant $N>0$, such that $\frac{\left\|f^{i}\right\|}{q^{i}}<N$ for any $i \in \mathbf{Z}$.

If (1) or (2) above is true, then $f$ is a diagonalizable linear map with all eigenvalues of modulus $q$.

Proof of Theorem 1.4. Take an element $g \in G$. Then $g^{*}\left[B_{g}\right]=\left[B_{g}\right]$ for some big R-divisor $B_{g}$ as an interior point in the pseudo-effective cone of $X$. Note that the pseudo-effective cone and nef cone of $X$ are invariant by $\operatorname{Aut}(X)$. By Lemma 2.3, we get (2) in Lemma 2.3. Applying Lemma 2.3 to the nef cone $(=C)$ of $X$, we obtain (1) in Lemma 2.3. So there is an ample R-divisor $H_{g}$ such that $g^{*}\left[H_{g}\right]=\left[H_{g}\right]$ and $g^{*}$ is a diagonalizable map with all eigenvalues of modulus 1. By [15, Lemma 3.5], we may assume $H_{g}$ is an ample Cartier divisor. Take $L:=N_{\mathbf{Z}}^{1}(X) /$ (torsion). Let $D$ be a line bundle over $X$ and consider the polarization map

$$
\tau_{D}: \operatorname{Aut}(X) \rightarrow \operatorname{Pic}(X), g \longmapsto g^{*}(D) \otimes D^{-1}
$$

which takes the identity to the trivial bundle, and hence $\operatorname{Aut}_{0}(X)$ to $\operatorname{Pic}^{0}(X)$. This yields that $\left.\operatorname{Aut}_{0}(X)\right|_{L}=\{\mathrm{id}\}$. Let $r:=\operatorname{rank}(L)$. Notice that the characteristic polynomial $f(x)$ of $\left.g\right|_{L}$ is a monic polynomial of degree $r$ over $\mathbf{Z}$ whose all roots $\lambda$ are all of modulus 1. By Gauss's lemma, the minimal polynomial $p(x)$ of $\lambda$ has $p(x) \mid f(x)$ in $\mathbf{Z}[x]$. Then $p(x)$ is an irreducible monic polynomial over $\mathbf{Z}$ whose all roots have absolute value 1 and $\operatorname{deg}(p(x)) \leq r$. Thus, all $\lambda$ are all roots of unity by Kronecker's Theorem. So there is a minimal positive integer $d$ such that $\lambda^{d}=1$. This yields that $p(x)$ is a cyclotomic polynomial of degree $\varphi(d)$ and $\varphi(d) \leq r$. Here $\varphi(d):=\left|\operatorname{Gal}\left(\mathbf{Q}\left(\zeta_{d}\right) / \mathbf{Q}\right)\right|$ is the Euler function. There are finitely many $d$ with $\varphi(d) \leq r$. Let $m$ be their product, which is independent of $g$. Then every eigenvalue of $\left.g^{m}\right|_{L}$ is 1 . Moreover, $\left.g^{m}\right|_{L}=$ id since $\left.g\right|_{L}$ is diagonalizable. Take $\rho: G \rightarrow \operatorname{GL}\left(L \otimes_{\mathbf{Z}} \mathbf{C}\right)$, and ker $\rho:=G_{1}$. Now applying Burnside's theorem to $\operatorname{GL}\left(L \otimes_{\mathbf{Z}} \mathbf{C}\right)$, $\left.G\right|_{L}$ is finite and $\left[G: G_{1}\right]<\infty$. In particular, $\left.G_{1}\right|_{L}$ is trivial. This yields that $G_{1}$ fixes any ample class $[H]$ and then $G_{1} \leq \operatorname{Aut}_{[H]}(X)$. Thus $G_{1}$ (and hence $G$ ) is virtually in $\operatorname{Aut}_{0}(X)$ by [16, Remark 2.6]. This completes the proof of Theorem 1.4.

The following lemma implies Theorem 1.2(1).

Lemma 2.4. (cf. [6, Lemma 2.8]) Let $X$ be a projective variety of dimension $n$. Let $G$ be a subgroup of $\operatorname{Aut}(X)$ with only zero entropy elements. Then there is a finite-index subgroup $G^{\prime}$ of $G$ satisfying the following properties for every $1 \leq k \leq n-1$. 
(1) The kernel of the canonical representation

$$
\rho_{k}: G^{\prime} \longrightarrow \mathrm{GL}\left(N_{\mathbf{R}}^{k}(X)\right)
$$

is equal to $G_{0}^{\prime}$;

(2) The image of $\rho_{k}$ is a unipotent subgroup of $\mathrm{GL}\left(N_{\mathbf{R}}^{k}(X)\right)$.

Proof. By Remark 1.3(1) and Theorem 1.4, the proof of Lemma 2.4(1) is the same as [6, Lemma 2.8]. Here, we give another proof of Lemma 2.4(2), which is slightly different from [6, Proof of Lemma 2.8(2)]. We define $k$-th dynamical degree (see eg. [12, §2.4]) by the natural pullback $g^{*}$ on $N_{\mathbf{R}}^{k}(X)$ for any integer $0 \leq k \leq n$. Namely,

$$
d_{k}(g):=\rho\left(\left.g^{*}\right|_{N_{\mathbf{R}}^{k}(X)}\right)=\max \left\{|\lambda|: \lambda \text { is an eigenvalue of }\left.g^{*}\right|_{N_{\mathbf{R}}^{k}(X)}\right\} .
$$

Notice that $d_{1}(g) \leq 1$ since $g$ is of zero entropy. By the log-concavity of dynamical degrees (cf. [12, Corollary 2.11]), $d_{k}(g) \leq d_{1}^{k}(g)$ for every $k$. This yields that $d_{k}(g) \leq 1$ for every $k$. Let $r_{k}:=\operatorname{rank}\left(N_{\mathbf{R}}^{k}(X)\right)$. Notice that the characteristic polynomial $f_{k}(x)$ of $\left.g\right|_{N_{\mathbf{R}}^{k}(X)}$ is a monic polynomial of degree $r_{k}$ over Z Z. Hence, the product of all roots $\lambda_{k}$ of $f_{k}(x)$ is an integer. So all $\lambda_{k}$ are algebraic integers of modulus 1 as $d_{k}(x) \leq 1$. By Gauss's lemma, the minimal polynomial $p_{k}(x)$ of $\lambda_{k}$ has $p_{k}(x) \mid f_{k}(x)$ in $\mathbf{Z}[x]$. Then $p_{k}(x)$ is an irreducible monic polynomial over $\mathbf{Z}$ whose all roots have absolute value 1 and $\operatorname{deg}\left(p_{k}(x)\right) \leq r_{k}$. Thus, all $\lambda_{k}$ are all roots of unity by Kronecker's Theorem. So there is a minimal positive integer $d$ such that $\lambda_{k}^{d}=1$. This yields that $p_{k}(x)$ is a cyclotomic polynomial of degree $\varphi(d)$ and $\varphi(d) \leq r_{k}$. Here $\varphi(d):=\left|\operatorname{Gal}\left(\mathbf{Q}\left(\zeta_{d}\right) / \mathbf{Q}\right)\right|$ is the Euler function. There are finitely many $d$ with $\varphi(d) \leq r_{k}$. Let $m_{k}$ be their product, which is independent of $g$. So $\left.\left(g^{m_{k}}\right)\right|_{N_{\mathbf{R}}^{k}(X)}$ is unipotent. According to Lemma 2.2, replacing $G$ by a finite-index subgroup, we have that $\rho_{k}(G)$ contains only unipotent elements of $\operatorname{GL}\left(N_{\mathbf{R}}^{k}(X)\right)$. This completes the proof of Lemma 2.4(2).

Let $G \leq \operatorname{Aut}(X)$. We say that a rational map $f: X \rightarrow Y$ is $G$-equivariant or $f$ equivariantly, if there is a group homomorphism $\rho: G \rightarrow \operatorname{Aut}(Y)$ such that $f \circ g=\rho(g) \circ f$ for all $g \in G$. In [6, Lemma 2.9], the authors obtained a $G$-equivariant resolution by using a canonical resolution in [1, Theorem 13.2]. However, the existence of the resolution of singularities is not known in positive characteristic to the best of our knowledge. Note that the dynamical degrees in arbitrary characteristic is birational invariance (cf. [12, Lemma 2.8]). This motivates the following result, which allows us to work with suitable birationally equivalent models.

Proposition 2.5. (cf. [6, Lemmas 2.9 and 2.10]) Let $\pi: X_{1} \rightarrow X_{2}$ be a dominant rational map between projective varieties of the same dimension. Let $G$ be a group acting on both $X_{1}$ and $X_{2}$ biregularly and $\pi$-equivariantly. Then the following statements hold.

(1) Let $\widetilde{X_{1}}$ be the normalization of the graph of the map $\pi: X_{1} \rightarrow X_{2}$. Then there is a birational map $p_{1}: \widetilde{X_{1}} \rightarrow X_{1}$ such that $\pi \circ p_{1}: \widetilde{X_{1}} \rightarrow X_{2}$ is a G-equivairant 
surjective morphism. In particular, $p_{1}^{-1} \circ G \circ p_{1}$ is a subgroup of $\operatorname{Aut}\left(\widetilde{X_{1}}\right)$ and is isomorphic to $G$.

(2) Suppose $\left.G\right|_{X_{1}}$ (or equivalently $\left.G\right|_{X_{2}}$ ) is of zero entropy. Then we have

$$
\ell_{\mathrm{ess}}\left(\left.G\right|_{X_{1}}, X_{1}\right)=\ell_{\mathrm{ess}}\left(\left.G\right|_{X_{2}}, X_{2}\right) .
$$

Further, replacing G by a finite-index subgroup, $\left(\left.G\right|_{X_{1}}\right) /\left(\left.G\right|_{X_{1}}\right)_{0} \cong\left(\left.G\right|_{X_{2}}\right) /\left(\left.G\right|_{X_{2}}\right)_{0}$.

Remark 2.6. The proof of Proposition 2.5(1) is the same as [6, Proof of Lemma 2.9] without using the canonical resolution in [1, Theorem 13.2]. After replacing $H^{1,1}(X, \mathbf{R})$ by $N_{\mathbf{R}}^{1}(X)$, the proof of Proposition 2.5(2) is the same as [6, Lemma 2.10] by using [12, Lemma 2.8].

Chevalley's theorem on algebraic groups asserts that every algebraic group over a perfect field is 'built up' from a linear algebraic group and an abelian variety. By Chevalley's theorem and [22, Theorem 14.1], if a complex projective variety $X$ is not uniruled, then $\operatorname{Aut}_{0}(X)$ is an abelian variety. This result is also known in positive characteristic as follows.

Lemma 2.7. (cf. [2, Proposition 7.1.4]) Let $X$ be a projective normal variety which is not uniruled. Then $\operatorname{Aut}_{0}(X)$ is an abelian variety.

The following Proposition 2.8 implies Theorem 1.2(2) by Lemmas 2.1 and 2.4.

Proposition 2.8. Let $X$ and $G$ be as in Theorem 1.2. Then we have

$$
\ell_{\mathrm{ess}}(G, X) \leq n-1
$$

Remark 2.9. By Remark 1.3(1), the proof of Proposition 2.8 uses the same argument as [6, Proposition 3.1].

Corollary 2.10. ( $c f$. [6, Corollary 3.2 and Proposition 5.5]) Let $X$ be a projective normal variety of dimension $n \geq 1$. Let $G \leq \operatorname{Aut}(X)$ be a group of zero entropy automorphisms. Suppose that $\operatorname{Aut}_{0}(X)$ is commutative (for instance, this holds when $X$ is not uniruled, see Lemma 2.7). Then

(1) We have the following (in)equalities

$$
\ell_{\min }(G) \leq \ell_{\min }\left(G / G_{0}\right)+1=\ell_{\min }\left(\left.G\right|_{N_{\mathbf{R}}^{1}(X)}\right)+1=\ell_{\mathrm{ess}}(G, X)+1 \leq n .
$$

(2) If $\ell_{\min }(G)=n$, then we have

$$
G_{0} \neq\{1\} \quad \text { and } \quad \ell_{\mathrm{ess}}(G, X)=n-1
$$

Remark 2.11. After replacing $H^{1,1}(X, \mathbf{R})$ by $N_{\mathbf{R}}^{1}(X)$, the proof of Corollary 2.10 is the same as [6, Corollary 3.2]. 


\section{ON THE NILPOTENCY CLASS OF ZERO ENTROPY GROUPS}

For a group $G$ and a non-negative integer $c$, the lower center series $G_{(c)}$ is defined inductively by

$$
G_{(0)}:=G \quad \text { and } \quad G_{(i+1)}:=\left[G_{(i)}, G\right] .
$$

By definition, $G_{(c)}=\{1\}$ for some non-negative integer $c$ exactly when $G$ is nilpotent. We call the minimum of such $c$ the nilpotency class of $G$ (when $G$ is nilpotent) and denote it by $\mathfrak{c}(G)$. A normal series $G=G_{1} \triangleright G_{2} \triangleright \cdots \triangleright G_{n}=1$ with each $G_{i} \unlhd G$ and $G_{i} / G_{i+1} \leq Z\left(G / G_{i+1}\right)$ is a central series. It is well known that a group $G$ is nilpotent if and only if it has a central series $G=G_{1} \triangleright G_{2} \triangleright \cdots \triangleright G_{n}=1$.

Lemma 3.1. (cf. [18, Lemma 2.1]) Let $V$ be a positive dimensional vector space and $G$ a nilpotent (or solvable) subgroup of $\mathrm{GL}(V)$. Then:

(1) Any subgroup of $G$ and any quotient group of $G$ are nilpotent (or solvable).

(2) The Zariski closure $\bar{G}$ of $G$ in $\mathrm{GL}(V)$ is also nilpotent (or solvable).

Proof. When $G$ is solvable, this lemma is [18, Lemma 2.1]. We only show (2) when $G$ is nilpotent. It suffices to check that $\overline{[H, G]}=[\bar{H}, \bar{G}]$ for $H \unlhd G$. Note that $[\bar{H}, \bar{G}]$ is closed in $\bar{G}$ (see for instance $[13, \S 17.2]$ ). Thus $\overline{[H, G]} \subset[\bar{H}, \bar{G}]$.

Let us show the other inclusion. Take $g \in G$. Let us define the map $\theta_{g}$ by

$$
\alpha_{g}: \bar{H} \rightarrow \overline{[H, G]} ; g \mapsto f^{-1} g^{-1} f g .
$$

Clearly, $\alpha_{g}$ is continuous and satisfies $\alpha_{g}(H) \subset[H, G]$. Thus, $\alpha_{g}(\bar{H}) \subset \overline{[H, G]}$. Hence, $[\bar{H}, G] \subset \overline{[H, G]}$. Let $f \in \bar{H}$. Let us define the map $\beta_{f}$ by

$$
\beta_{f}: \bar{G} \rightarrow \bar{G} ; g \mapsto f^{-1} g^{-1} f g .
$$

Clearly, $\beta_{f}$ is continuous and satisfies $\beta_{f}(G) \subset[\bar{H}, G]$. Since $[\bar{H}, G] \subset \overline{[H, G]}$, we have $\beta_{f}(G) \subset \overline{[H, G]}$ as well. Thus, $\beta_{f}(\bar{G}) \subset \overline{[H, G]}$ and hence $[\bar{H}, \bar{G}] \subset \overline{[H, G]}$.

Remark 3.2. In the proof of Proposition 2.8, the derived series of zero entropy groups follows from the abelian quotient groups $H_{i+1} / H_{i}$ as in [6]. Note that some lemmas in the proof of Proposition 2.8 are also true after replacing the derived series by the lower center series. For instance, $\mathfrak{c}\left(G^{\prime} / G_{0}^{\prime}\right)$ does not depend on the choice of $G^{\prime}$, where $G^{\prime}$ is a finite-index subgroup of $G$ as in Theorem 1.2 such that $G^{\prime} / G_{0}^{\prime}$ is a unipotent group. Using Lemma 3.1, this proof is similar as Lemma 2.1. Observe that it is hard to figure out a crucial result (which is similar to [6, Proposition 2.6]) for constructing a central series $G_{i}$ such that $G_{i} / G_{i+1} \leq Z\left(G / G_{i+1}\right)$. These raise the following question.

Question 3.3. Let $X$ be a projective variety of dimension $n \geq 1$ and $G$ a subgroup of $\operatorname{Aut}(X)$ such that all elements of $G$ have zero entropy. Let $G^{\prime}$ be a finite-index subgroup of $G$ such that $G^{\prime} / G_{0}^{\prime}$ is a unipotent group. Is there a nonnegative integer $c$ such that $\mathfrak{c}\left(G^{\prime} / G_{0}^{\prime}\right)$ is at most equal to $c$ ? Moreover, is $c=n-1$ right? 
For Question 3.3, we quote the following result in [20], which is noticed by Fei Hu.

Proposition 3.4. ( $c f .[20,5.1 .12])$ Let $G$ be a nilpotent group. Then $G$ is solvable, and we have:

$$
\ell(G) \leq \log _{2} \mathfrak{c}(G)+1
$$

\section{ACKNOWLEDGMENTS}

The author gratefully acknowledge De-Qi Zhang for many inspiring discussions and comments. He would like to thank Fei Hu and Guolei Zhong for helpful communications and the anonymous referee for several suggestions.

\section{REFERENCES}

[1] E. Bierstone and P. D. Milman, Canoncial desingularization in characteristic zero by blowing up the maximum strata of a local invariant, Invent. Math. 128 (1997), 207-302. $\uparrow$ 6, 7

[2] M. Brion, Some Structure Theorems for Algebraic Groups, Algebraic Groups: Structure and Actions, Proc. Sympos. Pure Math., 94, American Mathematical Society. Providence, pp.53126(2017). $\uparrow 1,7$

[3] F. Campana, F. Wang and D.-Q. Zhang. Automorphism groups of positive entropy on projective threefolds, Trans. Amer. Math. Soc. 366 (2014), no. 3, 1621-1638. $\uparrow 2$

[4] N. B. Dang, Degrees of iterates of rational maps on normal projective varieties, arXiv: $1701.07760 . \uparrow 2$

[5] T.-C.Dinh, F. Hu and D.-Q. Zhang, Compact Kähler manifolds admitting large solvable groups of automorphism, Adv. Math. 281 (2015), 333-352. $\uparrow 3$

[6] T.-C. Dinh, K. Oguiso and D.-Q. Zhang, Derived length of zero entropy groups acting on compact Kähler manfiolds, arXiv: 1810 .04827v1. $3,4,5,6,7,8$

[7] T.-C. Dinh and N. Sibony, Groupes commutatifs d'automorphismes d'une variété kählérienne compacte, Duke Math. J. 123 (2004), no. 2, 311-328. 22

[8] T.-C. Dinh and N. Sibony, Equidistribution problems in complex dynamics of higher dimension, Internat. J. Math. 28 (2017), no. 7, 1750057, 31 pp. $\uparrow 2$

[9] A. Fujiki, On automorphism groups of compact Kähler manifold, Invent. Math. 44 (1978), 225258. $\uparrow 3$

[10] W. Fulton, Intersection Theory, 2nd ed., Springer, Berlin, 1998. $\uparrow 4$

[11] F. Hu and S. Li, Free abelian group actions on normal projective varieties: sub-maximal dynamical rank case, arXiv:1907.00229. $\uparrow 2$

[12] F. Hu, A theorem of Tits type for automorphism groups of projective varieties in arbitrary characteristic (with an appendix by Tomohide Terasoma), to appear in Math. Ann., 30 pp., DOI:10.1007/s00208-019-01812-9, arXiv:1801.06555.个2,3,6, 7

[13] J. E. Humphreys, Linear algebraic groups, Graduate Text in Mathematics, vol. 21, SpringerVerlag, New York-Heideberg, 1975. $\uparrow 3,8$

[14] D. I. Lieberman, Compactness of the Chow scheme: applications to automorphisms and deformations of Kähler manifolds. Fnctions de plusieurs varibales complexes, III (Sém. François Norguet, 1975-1977), pp. 140-186, Lecture Notes in Math. vol. 670, Springer, Berlin, 1978. 3

[15] S. Meng and D.-Q. Zhang, Building blocks of polarized endomorphisms of normal projective varieties, Adv. Math. 325 (2018), 243-273. $\uparrow 3,4,5$ 
[16] S. Meng and D.-Q. Zhang, Jordan property for non-linear algebraic groups and projective varieties, Amer. J. Math. 140 (2018), 1133-1145. $\uparrow, 5$

[17] N. Nakayama and D.-Q. Zhang, Building blocks of étale endomorphisms of complex projective manifolds, Proc. Lond. Math. Soc. 99 (2009), 725-756. 33

[18] K. Oguiso, Tits alternative in hyperkähler manifolds, Math. Res. Lett. 13 (2006), 307-316. $\uparrow 8$

[19] Z. Patakfalvi, On subadditivity of Kodaira dimension in positive characteristic over a general type base, J. Algebr. Geom. 27(1)(2018), 21-53. $\uparrow 2$

[20] D. J. S. Robinson, A Course in the theory of groups, Second edition, Graduate Text in Mathematics, vol. 80, Springer-Verlag, New York, 1996. $\uparrow 8$

[21] T. T. Truong, Relative dynamical degrees of correspondences over a field of arbitrary characteristic, to appear in J. reine angew. Math.,DOI:10.1515/crelle-2017-0052, arXiv:1605.05049. 2

[22] K. Ueno, Classification theory of algebraic varieties and compact complex spaces, Lecture Notes in Mathematics, vol. 439, Springer-Verlag, Berlin-New York, 1975, Notes written in collaboration with P. Cherenack. $\uparrow 7$

[23] D.-Q. Zhang, A theorem of Tits type for compact Kähler manifolds, Invent. Math. 176 (2009), no. 3, 449-459. $\uparrow 2$

[24] D.-Q. Zhang, $n$-dimensional projective varieties with the action of an abelian group of rank $n-1$, Trans. Amer. Math. Soc. 368 (2016), no. 12, 8849-8872. $\uparrow 2$

School of Mathematical Sciences, Fudan University, Shanghai 200433, People's RepubLIC OF CHINA

Department of Mathematics, National University of Singapore, Block S17, 10 Lower KENT Ridge RoAd, Singapore 119076

E-mail address: lisichen123efoxmail.com

URL: https://www.researchgate.net/profile/Sichen_Li4 